\title{
Information Management in Cancer Registries: Evaluating the Needs for Cancer Data Collection and Cancer Research
}

Iris Zachary ${ }^{1,2,3 *}$, Suzanne A Boren ${ }^{1,2}$, Eduardo Simoes ${ }^{1,2,3}$, Jeannette Jackson-Thompson ${ }^{1,2,3}$,

J Wade Davis ${ }^{1,2}$, Lanis Hicks ${ }^{1,2}$

1. Department of Health Management and Informatics, School of Medicine, University of Missouri, Columbia, Missouri

2. MU Informatics Institute, University of Missouri, Columbia, Missouri

3. Missouri Cancer Registry and Research Center, Health Management and Informatics, School of Medicine, University of Missouri, Columbia, Missouri

\begin{abstract}
Cancer registry data collection involves, at a minimum, collecting data on demographics, tumor characteristics, and treatment. A common, identified, and standardized set of data elements is needed to share data quickly and efficiently with consumers of this data. This project highlights the fact that, there is a need to develop common data elements; Surveys were developed for central cancer registries (CCRs) and cancer researchers (CRs) at NCl-designated Cancer Centers, in order to understand data needs. Survey questions were developed based on the project focus, an evaluation of the research registries and database responses, and systematic review of the literature. Questions covered the following topics: 1) Research, 2) Data collection, 3) Database/ repository, 4) Use of data, 5) Additional data items, 6) Data requests, 7) New data fields, and 8) Cancer registry data set. A review of the surveys indicates that all cancer registries' data are used for public health surveillance, and $96 \%$ of the registries indicate the data are also used for research. Data are available online in interactive tables from over $50 \%$ of CRs and $87 \%$ of CCRs. Some other survey responses indicate that CCR treatment data are not complete for example treatment data, however cancer researchers are interested in treatment variables from CCRs. Cancer registries have many data available for review, but need to examine what data are needed and used by different entities. Cancer Registries can further enhance usage through collaborations and partnerships to connect common interests in the data by making registries visible and accessible.
\end{abstract}

Keywords: Public Health; Disease Registries; Disease Reporting

Correspondence: zacharyi@missouri.edu

DOI: 10.5210/ojphi.v7i2.5664

Copyright (C2015 the author(s)

This is an Open Access article. Authors own copyright of their articles appearing in the Online Journal of Public Health Informatics. Readers may copy articles without permission of the copyright owner(s), as long as the author and OJPHI are acknowledged in the copy and the copy is used for educational, not-for-profit purposes 


\section{Introduction}

Cancer registries are information systems designed for the collection, management, storage, and analysis of data on individuals diagnosed with cancer [1]. Cancer registries collect data elements that include demographics, diagnoses, tumor histology, treatment, and outcome information [2]. There are three types of cancer registries: 1) facility-based registries that collect information about patients diagnosed and/or treated at that facility, 2) specialty registries that collect information on one type of cancer (e.g., brain tumors, pediatric cancers) and 3) central cancer registries (CCRs) that collect information about cancer patients in a particular geographic area (e.g., a region, state or territory) [3-5]. Cancer registries have a long history, with the first registries going back to the early 1900s when individual physicians or institutions formed case-based cancer registries [1]. The first hospital cancer registry was established at Yale in New Haven, Connecticut in 1926. The first CCRs were established in 1935 in Connecticut and 1946 in California. Public Law 92-218, the National Cancer Act of 1971, directed National Cancer Institute (NCI) to "collect, analyze, and disseminate all data useful in the prevention, diagnosis, and treatment of cancer." [6] This led to the establishment of the Surveillance, Epidemiology and End Results (SEER) program in 1973. The first national cancer registry in the United States, the SEER Program covered only a few states, representing only about $10 \%$ of the total US population. Congress established the National Program of Cancer Registries (NPCR) (Public Law 102-515) in 1992 to be administered by the CDC [7]; the CDC's charge was to establish a Central Cancer Registry (CCR) in states without a registry and enhance registries in states with an existing registry, thus covering the entire nation. High-quality cancer incidence data are available for over $96 \%$ of the US population as a result of these public laws. SEER and CDC-NPCR, along with the Commission on Cancer (CoC), are standard setters for cancer registries. Other groups that work with and make recommendations to standard setters are the American Joint Committee on Cancer (AJCC), the North American Association of Central Cancer Registries (NAACCR), the American Cancer Society (ACS), the National Cancer Registrars Association (NCRA) and the World Health Organization (WHO) [6].

Cancer data collection and analysis is required, but only complete, accurate, and timely data can make a significant difference in the field of cancer research and further efforts in the evaluation, surveillance and prevention of cancer [8,9]. Parkin (2008) emphasizes that the role of cancer registries has expanded in the last two decades to include not only collection of cancer diagnosis and treatment data, but also planning and evaluation of cancer control activities, involvement in patient care and survival data. The expansion of cancer registry data collection will become an important factor in the global fight against cancer [10-12].

Furthermore, cancer registration is a continuously changing field that is heavily impacted by the rapidly advancing medical field, expansion of the medical knowledge base, and coding of disease and treatment [10,13-15]. Being responsive to these changes often means that data fields are added or modified, making accurate and complete data collection even more challenging. The standardization of cancer registry data is an important part of cancer registration, and registries have expanded from approximately 25 required data elements to more than 200 required data elements within the last two decades. However, by continuously adding data elements, data collection may be getting too specific and consequently lacking in completeness. Evaluation, surveillance, and prevention of cancer rely on the statistics that are obtained from cancer registry data. Data in the 
CCR are necessary to support public health efforts and to provide surveillance studies that give us information on long-term effects of cancer. This project examines the theory that, among the data elements currently collected by registries, there is a shared utility of certain elements for CCR $\underline{\text { and }}$ registry data consumers; the project further theorizes that a collection of these core, meaningful data elements could help further the mission of these CCRs and better serve consumers. The project also attempts to identify problems with data collection and aims to support the idea for development of a core dataset that can better meet public health surveillance and research requirements. The overarching question is: What data elements are needed for public health surveillance, and what data elements are needed for research will need to be addressed in detail in a future study.

\section{Methods}

\section{Survey Development and Content}

Investigators developed two surveys, one for the CCRs and one for cancer researchers, with input from experts in the field of cancer registry content, survey research, health information technology, and cancer research. Based on the focus of the research, the evaluation of the research registries and databases, and systematic review of the literature, the survey was developed and pilot tested. The pilot survey was sent to five internal and ten external participants. Five of the internal and six of the external participants responded and gave suggestions for clarification of some of the questions in the surveys. The surveys pose questions about the data elements essential to the cancer research community, barriers to data use, and necessity of data for the cancer registry and the cancer researcher. The survey content was reviewed internally at the Missouri Cancer Registry and Research Center and approved by the Health Science Institutional Review Board at the University of Missouri. The survey instrument for CCRs contained 41 questions on eight topics: 1) Research, 2) Data collection, 3) Database/ repository, 4) Use of data, 5) Additional data items, 6) Data requests, 7) New data fields, and 8) Cancer registry dataset. The survey instrument for researchers contained 32 questions on topics 1 through 6. The 50 state CCRs as well as the District of Columbia comprised one core group. Forty-three out of 51 registries were represented. Another core group included researchers at NCI-designated Comprehensive Cancer Centers (41) and National Cancer Institute Cancer Centers (26). There was no randomization because of the small sample size.

The two surveys were administered simultaneously between October 2011 and March 2012. Both groups received invitation by email to participate in the web based surveys. The participants were provided with a web link to access the surveys hosted on Survey Monkey. Non-responders received two reminders each after four weeks. In February 2012, the survey was also sent to two scientific working groups (AMIA CRI-WG and AMIA PHI-WG) to expose the survey instrument to a broader audience. The survey was closed in May 2012. Data were collected and checked for errors, such as missing data, as well as editing and data entry errors. Descriptive statistics, including the total number and the geographic areas of the respondents, describing the groups that were studied are presented in this section. The descriptive statistics give information about the specific groups that are studied but cannot be generalized to any larger group due to size. In total, 43 cancer registry personnel responded to the Central Cancer Registry Survey and 28 responded to the Cancer Research Survey. Of the responders, 35 (88\%) were from NPCR registries, four (10\%) were from SEER registries and one (2\%) was a SEER- and NPCR-funded registry. The response rates to questions from NCI-designated research centers were more limited (43\%). Survey answers that included "not sure" or "don't know" were included in the response count $[16,17]$. Additionally for 
this study, the project coordinators calculated the average based on the number of people who responded to each question. Each question was not answered by each respondent. None of the questions were required to be answered, some questions allowed multiple answers.

\section{Results}

A summary of the survey results identifies common use of data elements between cancer registries and researchers. Findings from the survey identify data elements that are necessary for research, such as surveillance, and data collection, and did help to clarify some barriers to the use of cancer registry data. Based on the review of the answers, it is apparent from the two surveys that a gap exists between the data elements collected and the data elements needed for surveillance and research. The data elements required for public health surveillance and research do overlap, (e.g. date of diagnosis, diagnosis, site, histology), however they are needed for different reasons. For example, the date of diagnosis is important for any longitudinal or public health-related study, while the date of diagnosis can also be needed by the researcher in order to determine if a patient could be included in studies relating to treatment, follow up or possible trial studies.

\section{Central Cancer Registry Survey}

The overall response for CCRs was 43 out of 51 surveys (84.3\%). The respondents were evenly distributed over the United States: 11 (27\%) from the Northeast, 12 (29\%) from the Midwest, 10 (24\%) from the South and eight (20\%) from the West.

Thirty-five (88\%) respondents are funded by NPCR, four (10\%) are funded by SEER, and one registry is funded by both NPCR and SEER. Twenty-three (66\%) registries are located at a State Health Department and 12 (34\%) are located at Universities.

Table 1 Cancer Registry Region and Location

\begin{tabular}{llc}
\hline Survey Item & & $\begin{array}{c}\text { Response } \\
(\mathrm{N}=41)\end{array}$ \\
\hline $\begin{array}{l}\text { In which geographic } \\
\text { registry located? }\end{array}$ region is your & \\
Northeast & $11(27)$ & \\
Midwest & $12(29)$ & \\
South & $10(24)$ & \\
West & $8(20)$ & $23(66)$ \\
Where is your registry located? & & $12(34)$ \\
State Health Department & & \\
University & 0 & \\
Not sure & & \\
\hline
\end{tabular}

*Not all respondents answered all questions

According to the survey results, registries have good data on demographics as well as stage, size and histology as a part of registry certification, but are lacking data on treatment variables. Additionally, respondents pointed out that accuracy and completeness is heavily dependent on site and histology 
even for certified registries. Capturing all cases can be challenging for sites that are treated outside the hospital setting, such as melanoma of the skin, prostate cancer, and blood disorders related to cancer. In one question, the registries were asked about the top five state- specific items collected in addition to the NAACCR/SEER required fields. Twelve (100\%) respondents collect tobacco, tobacco history, tobacco years, last name and first name, address at diagnosis, blood quantum, and one of the 12 respondents did not collect any state-specific items. When asked how many years of data the CCRs have available, 20 (87\%) respondents said that they had more than 10 years of data available, two (9\%) had between six and 10 years of data available, and only one respondent's registry has zero to five years of data available. Fifteen (68\%) respondents reported that they receive updated information on vital status and tumor status for each case. One respondent indicated that they receive vital status only through linkage with state vital records.

Table 2 shows the use of cancer registry data. Respondents were able to choose multiple answers in the survey process. When asked if the cancer registries could fill all data requests they receive, 15 (68\%) answered that they could not fill all data requests, six (27\%) answered that they could fill all data requests, and one (5\%) was not sure if they could fill all data requests.

Table 2 Cancer Registry Data Requests

\begin{tabular}{ll}
\hline Survey Item & Response (N=22) \\
\hline $\begin{array}{l}\text { Can you fill all data requests the cancer registry } \\
\text { receives? }\end{array}$ & \\
Yes & $6(27)$ \\
No & $15(68)$ \\
Not sure & $1(5)$ \\
$\begin{array}{l}\text { If you cannot fill all data requests, specify } \\
\text { the reason(s)? (Check all that apply) }\end{array}$ & \\
& $12 *$ \\
Data elements are not collected & 12 \\
Data elements are not available & 5 \\
$\begin{array}{l}\text { Data elements are not reliable } \\
\text { Data elements are not complete }\end{array}$ & 7 \\
Data elements have missing /unknown value & 8 \\
\hline
\end{tabular}

*Not all respondents answered all questions

* Data elements not complete include unknown and known values that may or may have been entered but are not complete; Data elements missing or unknown value is a value that is missing or unknown and cannot be entered because it is unknown

Survey participants who indicated they could not fill all data requests were asked to provide a reason. Twelve (100\%) replied one possible reason was that the data elements are not collected, five (42\%) said data elements requested are not available (for any reason), seven (58\%) said data elements are not reliable, eight (67\%) answered that data elements requested are not complete, and four (33\%) responded that the data elements have missing and unknown values. Several respondents mentioned that they do not have adequate staff to fill certain data requests or the necessary approval for the data requests may be unavailable. When asked how they felt about the number of data items required to be collected, 17 (77\%) indicated that too many were required, five (23\%) answered 
about right, none answered too few, and some respondents placed emphasis not on the number of data items but on type and quality of those items. When asked if they were interested in collecting additional data items, 19 (86\%) responded no and three (14\%) responded yes, pointing out that areas such as socio-economic factors, family history, genomic assays, tumor and bio markers are needed to keep up with developments in the field.

One respondent suggested that data collection for cancer registries has shifted due to the change in healthcare from inpatient to outpatient diagnosis and treatment of patients and the expanded role and additional work that registries are covering now that include many informatics and additional data collection activities. This brings up the issue of collecting data that is needed. This project asks if we should look at our datasets and explore the utility and use or need of data collected in the registry. Cancer registry respondents indicated that the data are used for public health surveillance (23; $100 \%)$, database linkages that include programs like Breast and Cervical Cancer Control Programs (22; 96\%), and research (21; 91\%), followed by cancer inquiries (20; 87\%), special projects (19; $83 \%)$, next-of-kin requests $(11 ; 48 \%)$, and clinical trials $(4 ; 17 \%)$. A few respondents named additional needs for information related to tissue repository of bio-specimens, FDA monitoring projects, and program planning. Cancer registry data are available online in interactive tables at the county level by 13 (59\%) CCRs. Researchers focus on treatment and need treatment variables for many studies, where the public health and surveillance focus is on the actual cancer diagnosis and follow up, as well as vital status to determine planning, survival and cancer control. The continuous addition of data elements by certifying agencies introduces the discussion of specificity versus completeness of the registry dataset. As mentioned earlier, data that are collected in registries are most beneficial for data analysis and research, public health, and surveillance when the data are accurate, timely, and complete. Eighty-seven percent (87\%) of cancer registry respondents have more than 10 years of population based data available for use.

Table 3 Cancer Registry Data and Data Availability

\begin{tabular}{|c|c|c|c|}
\hline Survey Item & $\begin{array}{l}\text { Response } \\
(\mathrm{N}=22)\end{array}$ & & \\
\hline & Yes & No & Not sure \\
\hline $\begin{array}{l}\text { Do you receive updated information on vital } \\
\text { status and tumor status for each case? }\end{array}$ & $15(68)$ & $7(32)$ & 0 \\
\hline $\begin{array}{l}\text { Do you make the cancer registry } \\
\text { data available for data requests? }\end{array}$ & $22(100)$ & 0 & N/A \\
\hline $\begin{array}{l}\text { Are registry data available online in } \\
\text { interactive tables? }\end{array}$ & $13(59)$ & $9(41)$ & N/A \\
\hline $\begin{array}{l}\text { Can you fill all data requests the } \\
\text { cancer registry receives? }\end{array}$ & $6(27)$ & $\begin{array}{c}15 \\
(68)\end{array}$ & $1(5)$ \\
\hline $\begin{array}{l}\text { Do you consider NAACCR silver } \\
\text { certification as research quality data?** }\end{array}$ & $14(70)$ & $6(30)$ & N/A \\
\hline
\end{tabular}


Are you interested in additional data items or elements that are not mentioned? If yes, in?

*Not all respondents answered all questions

**Silver certification meets: Case ascertainment has achieved $90 \%$ or higher completeness. A death certificate is the only source for identification of fewer than $5 \%$ of reported cancer cases. Less than $0.2 \%$ duplicate case reports are in the file. All data variables used to create incidence statistics by cancer type, sex, race, age, and county are 97\% error-free. Less than $3 \%$ of the case reports in the file are missing meaningful information on age, sex, county. Less than $5 \%$ of the cases in the file are missing meaningful information on race (US only).The file is submitted to NAACCR for evaluation within 23 months of the close of the diagnosis year under review.

\section{Cancer Research Survey}

We had 28 respondents for the cancer research survey of 66 NCI-designated cancer centers. The respondents were fairly evenly distributed over the United States, six (21\%) from the Northeast, nine (32\%) from the Midwest, seven (25\%) from the South and four (14\%) from the West. Eighteen (72\%) of the respondents are affiliated with a university or teaching hospital, five (20\%) with a hospital, two (8\%) with a physician group. Others included were a state health department, federal government agency, clinical research company or board of health.

Table 4 Cancer Research Region and Location

\begin{tabular}{lc}
\hline Survey Item & Response (N=26) \\
In which state is your facility located? & $9(32)$ \\
Northeast & $9(32)$ \\
Midwest & $7(25)$ \\
South & $4(14)$ \\
West & \\
Are you affiliated with? & $5(20)$ \\
Hospital & $18(72)$ \\
University/ Teaching Hospital & $2(8)$ \\
Physician Group & $2(8)$ \\
Not applicable & \\
\hline
\end{tabular}

*Not all respondents answered all questions

When asked how many years of data they have available in their database, the majority responded with greater than five years. The cancer researchers collect demographic data including race, ethnicity, and date of birth.

Table 5 Cancer Research Availability of Variables

\begin{tabular}{lll}
\hline Survey Item & $(\mathrm{N}=16)$ & Response
\end{tabular}




\begin{tabular}{|c|c|c|c|c|}
\hline $\begin{array}{l}\text { Which of the following } \\
\text { demographic data items do } \\
\text { vou collect? }\end{array}$ & & Yes & No & Not sure \\
\hline Name & 16 & $8(50)$ & $6(38)$ & $2(12)$ \\
\hline SSN & 16 & $6(38)$ & $8(50)$ & $2(12)$ \\
\hline Date of Birth & 16 & $12(75)$ & $3(19)$ & $1(6)$ \\
\hline Ethnicity & 16 & $12(75)$ & $3(19)$ & $1(6)$ \\
\hline Race & 16 & $12(75)$ & $3(19)$ & $1(6)$ \\
\hline Address at Diagnosis & 16 & $8(50)$ & $6(38)$ & $2(12)$ \\
\hline Current Address & 16 & $6(38)$ & $8(50)$ & $2(12)$ \\
\hline
\end{tabular}

*Not all respondents answered all questions

The group of CR respondents noted less than 5\% missing or unknown values in those fields, respectively; 50\% (eight) for date of birth; $46.7 \%$ (seven) and 33.3\% (five) for ethnicity in Figure 1.

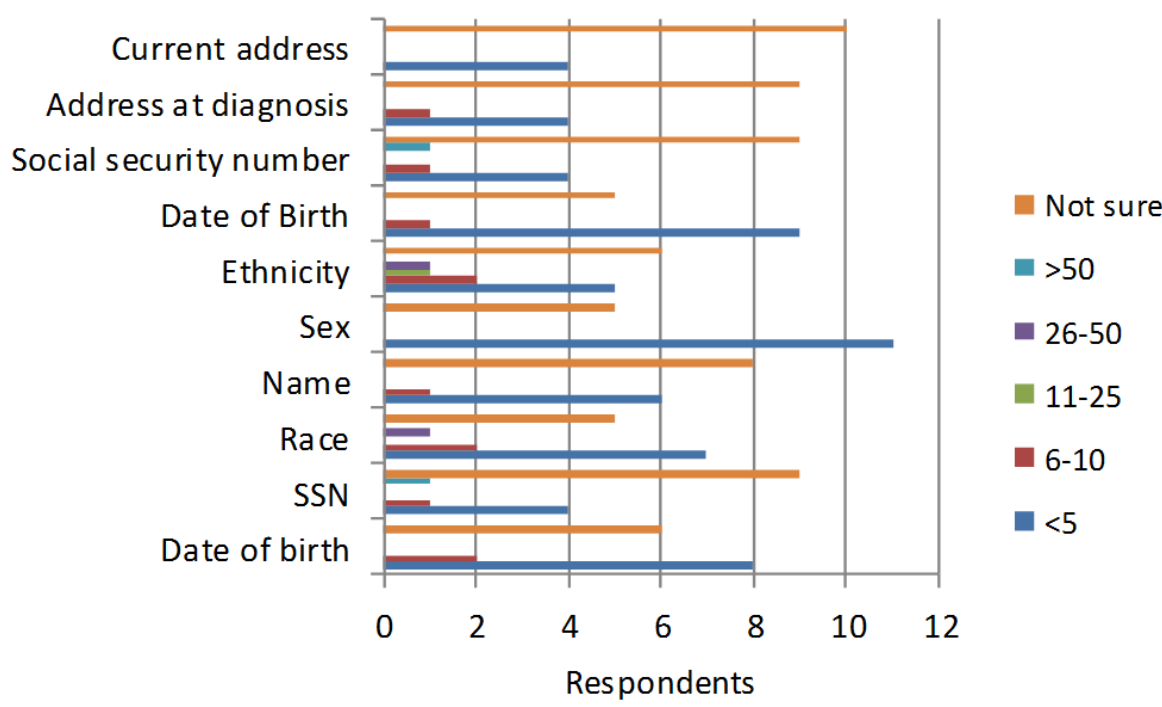

Figure 1: What percent of the variables have missing or unknown values?

When asked what other data elements or fields are of interest to them, the CR respondents listed treatment, staging, outcomes, diagnosis information, number of hospital stays, occupation and industry, comorbidity, family history, biomarkers, diet, exercise, treatment failure, AJCC staging, diagnostic evaluations and cause of death. A few researchers mentioned a preference for staging, diagnostic evaluations, and diagnostic information cancer registries can provide. Cancer research respondents were able to obtain information on tumor and treatment variables, but have missing or unknown values in both categories. The majority did not know how many data elements have missing or unknown values in these two categories. Eight (62\%) of the respondents have less than $5 \%$ missing or unknown values for the data element of tumor site.

\section{Table 6 Cancer Research Data}

\begin{tabular}{llcl}
\hline Survey Item & Response (N=14) & \\
\hline & Yes & No & Not
\end{tabular}


Do you collect information on vital status and tumor status?

Are your data complete for all fields in most of your studies?

Are the data standardized?

Are the data deduplicated?

Do you know what data elements are available from the state?

$\begin{array}{ccc} & & \text { sure } \\ 10(71) & 1(7) & 3(21) \\ & 3 & 1(9) \\ 7(63) & (27) & 0 \\ 11(92) & 1(8) & 0 \\ 8(67) & 2 & 2(17) \\ & (17) & \\ 8(67) & 2 & 2(17) \\ & (17) & \end{array}$

*Not all respondents answered all questions

\section{Discussion}

Data collected in the field of cancer registry are most useful for research, public health surveillance, evaluation, etc., when the data are accurate, timely, and complete $[7,8]$. The challenges to serve public health, surveillance and research include difficulties in collecting complete, accurate and timely data for all elements. Responders of the surveys pointed out some data elements that are essential for the cancer research community, as well as barriers and needs for the cancer registry and the cancer researcher. An important issue addressed was the continuous addition of new data elements to the required data set as required by national standards. For example, treatment fields are wanted, but other fields are added that may or may not provide the data elements that could support research. Therefore it is clear that some data needed are already collected but some data elements that are needed are not collected. Over 70 percent of CCRs agree that too many data elements are required, yet data requests often cannot be filled because researchers want data elements that are not collected or not available. More dialogue is needed with stakeholders to identify needs. Additionally, CCRs collect data fields that are state-specific and dependent on need for clinical research (i.e., special studies for a specific cancer site for a preset amount of time). CCRs have valuable data that should be available and accessible, not only for public health and surveillance, but also for research. Cancer registries have some of the data readily available for use that are needed and wanted by cancer researchers that include for example date of diagnosis, histology and cancer site. Continuous collaborations and new partnerships can be beneficial to the cancer registries and to the cancer researchers. Respondents of the CRs survey specifically asked for several data elements that are currently available from the cancer registries. Some of the named fields (e.g., treatment, staging, outcomes, diagnosis information, number of hospital stays, occupation and industry, comorbidity, family history, biomarkers, diet, exercise, treatment failure, AJCC staging, diagnostic evaluations and cause of death addition) are available in some hospital and cancer registries as part of special studies, others such as diagnosis information or cause of death addition are readily available. In addition do cancer registries with new developments in gene discoveries, tumor, and biomarkers have to rethink their collection and dataset? Can cancer registries become new leaders by working hand in hand for example with the bioinformatics laboratories in forming partnerships that rely on data exchange for necessary research and work in the cancer field? The gap and challenge of meeting the needs of public health, surveillance, and research for the future opens up new opportunities for new collaborations and partnerships for health and bioinformatics. 


\section{Recommendations}

Most CCRs have complete, timely, and accurate data for all the fields that are required by standard setters, but may lack other variables important to research. A possible solution is that population based registries focus on making a much smaller minimum core data set available that is timely, accurate, and complete and fills the need for public health and surveillance and also accommodates some additional data elements useful for research. Further studies are needed to determine what is needed in a minimum dataset that fulfills the mission of a registry dataset and yet allows for collection of special data elements that are specifically interesting to researchers. Analysis to determine what data fields are mostly used for public health and surveillance are needed to determine a minimum core dataset.

\section{Limitations}

The limitations of this study are the small group of participants for the pool of central cancer registries (e.g. one central registry per state). Further, participants of the NCI designated cancer centers were not always directly involved in research. This resulted in a much lower response rate for the participant group. Because of the small population, only limited results could be included to maintain anonymity of the registries. Because the surveys were planned for researchers and cancer registries, the questions had to be somewhat similar to be comparable. This resulted in less specified questions. The research was to understand and identify views of both groups on the utilization and availability of data collected on cancer. Additional research is necessary to identify data needs and use for both groups.

\section{Conclusions}

Survey responses indicate that a more in-depth study is warranted to determine registry and researcher needs and use of data elements. Furthermore, the role of CCRs is expanding with advancements in genomics, tumor marker information that is dependent on site, pathways and predictive markers that have prognostic significance. With those advancements CCRs will play important roles for cancer surveillance, cancer control, and research. Additionally this information and change in the type of data, the amount of data, the importance of data fields for these specific purposes needs to be considered when developing a core data set for registries. The CCRs will be holding a wealth of information that pertains to cancer diagnosis, origin of cancer, and treatment. CCRs can become the information broker but also information holder for the purpose of public health, surveillance and research all at the same time.

\section{References}

1. Training Modules SEER. Brief History of Cancer Registration. U. S. National Institutes of Health, National Cancer Institute. 10/15/2012 http://training.seer.cancer.gov/

2. Wingo PA. 2005. A national framework for cancer surveillance in the United States. Cancer Causes Control. 16, 151-70. PubMed http://dx.doi.org/10.1007/s10552-004-3487-5

3. Menck H, Smart C, eds. Central Cancer. Registries, Design, Management, and Use. (Hargood Academic Publishers, Switzerland, 1994). 
4. Menck HR, Gress DM, Griffin A, Mulvihill L, Hofferkamp J, et al. Cancer Registry Management: Principles and Practices for Hospital and Central Registries. Third Edition. Dubuque, Iowa: Kendall Hunt; 2011.

5. Austin DF. Cancer registries: A tool in epidemiology. Rev Cancer Epidemiol, ed. Lilienfeld AM, Elsevier North-Holland, Pub, NY, NY 2:119-140, 1983.

6. Havener L, Thornton M, eds. Standards for Cancer Registries Volume II: Data Standards and Data Dictionary, Thirteenth Edition, Version 11.3. Springfield, IL: North American Association of Central Cancer Registries, April 2008.

7. Bray F, Parkin DM. 2009. Evaluation of data quality in the cancer registry: Principles and methods. Part I: Comparability, validity and timeliness. Eur J Cancer. 45(5), 747-55. PubMed http://dx.doi.org/10.1016/j.ejca.2008.11.032

8. Das A. 2009. Cancer registry databases: an overview of techniques of statistical analysis and impact on cancer epidemiology. Methods Mol Biol. 471, 31-49. PubMed

9. Volkers N. 1993. National Statistics Source Reaches 20th Anniversary. J Natl Cancer Inst. 85(23), $\cdots$. PubMed

10. Parkin DM. 2008. The role of cancer registries in cancer control. Int J Clin Oncol. 13(2), 10211. PubMed http://dx.doi.org/10.1007/s10147-008-0762-6

11. Wagner G. History of cancer registration. In: Jensen OM, Parkin DM, Maclennan R, Muir CS, Skeet RG, eds. Cancer registration, principles and methods. Lyons: International Agency for Research on Cancer, 1991:3-6. (IARC Scientific Publication No 95.)

12. Heinze O, Ihls A, Bergh B. Development of an open source provider and organization registry service for regional health networks. HEALTHINF 2010 - 3rd International Conference on Health Informatics, Proceedings, p 535-537, 2010.

13. Parkin DM. 2006. The evolution of the population-based cancer registry. Nat Rev Cancer. 6(8), 603-12. PubMed http://dx.doi.org/10.1038/nrc1948

14. Bray F, Moller B. 2006. Predicting the future burden of cancer. Nat Rev Cancer. 6, 63-74. PubMed http://dx.doi.org/10.1038/nrc1781

15. Biancioni F, Brunori V, Valigi P, Stracci F, LaRosa F. Cancer registry and information technology: a new management system for integrating cancer registry and oncology departments. 2010 IEEE Workshop on Health Care Management, WHCM 2010.

16. Salant P, Dillman DA. (1994) How to conduct your own survey. New York: Wiley.

17. Sorra J, Franklin M, Streagle S. Survey User's Guide: Hospital Survey on Patient Safety Culture. AHRQ Publication No. 08-0060, September 2008. Agency for Healthcare Research and Quality, Rockville, MD. http://www.ahrq.gov/qual/nhsurvey08/nhguide.htm 